\title{
Generalized Albanese morphisms
}

\author{
Georg Hein
}

\begin{abstract}
We define nef line bundles $\mathcal{L}_{r}$ on a projective variety $X$ with the property that, for a curve $C \subset X$, the intersection $\mathcal{L}_{r} . C$ is zero, if and only if the restriction morphism $\operatorname{Hom}\left(\pi_{1}(X), \mathrm{U}(r)\right) \rightarrow \operatorname{Hom}\left(\pi_{1}(C), \mathrm{U}(r)\right)$ has finite image up to conjugation. This yields a rational morphism $X \stackrel{\operatorname{alb}_{r}}{\rightarrow} \operatorname{Alb}_{r}(X)$ contracting those curves $C$ with $\mathcal{L}_{r} . C=0$. For $r=1$ this is the Stein factorization of the Albanese morphism.
\end{abstract}

\section{Introduction}

The Albanese morphism $X \rightarrow \operatorname{Alb}(X)$ of a complex algebraic variety $X$ can be characterized in different ways. The Stein factorization $\mathrm{alb}_{1}: X \rightarrow \mathrm{Alb}_{1}(X)$ of this morphism can be described by those curves $C \subset Y$ which are contracted by alb 1 (see Proposition 2.4). Of interest to us is the following equivalence: a curve $\iota: C \rightarrow X$ is contracted by alb 1 , if and only if the restriction morphism $\operatorname{Hom}\left(\pi_{1}(X), \mathrm{U}(1)\right) \rightarrow \operatorname{Hom}\left(\pi_{1}(C), \mathrm{U}(1)\right)$ has finite image. A natural generalization would be a morphism $\operatorname{alb}_{r}: X \rightarrow \operatorname{Alb}_{r}(X)$ with the following property:

$$
\left(\begin{array}{c}
\text { a curve } \iota: C \rightarrow X \\
\text { is contracted by } \mathrm{alb}_{r}
\end{array}\right) \Leftrightarrow\left(\begin{array}{c}
\text { the restriction morphism } \\
\operatorname{Hom}\left(\pi_{1}(X), \mathrm{U}(r)\right) \rightarrow \operatorname{Hom}\left(\pi_{1}(C), \mathrm{U}(r)\right) \\
\text { has finite image modulo conjugation }
\end{array}\right) \text {. }
$$

The aim of this paper is to give, at least birationally, such a morphism. Suppose the generalized Albanese morphism with the above property existed. Take an ample divisor class $H_{r}$ on $\operatorname{Alb}_{r}(X)$. Define $\mathcal{L}_{r}$ to be the pull back of the ample line bundle $\mathcal{O}_{\operatorname{Alb}_{r}(X)}\left(H_{r}\right)$ to $X$. Eventually, we would obtain a nef line bundle $\mathcal{L}_{r}$ on $X$ fulfilling the following:

$$
\left(\begin{array}{c}
\text { for a curve } C \rightarrow X \\
\mathcal{L}_{r} . C=0 \text { holds }
\end{array}\right) \Leftrightarrow\left(\begin{array}{c}
\operatorname{the~restriction~morphism~} \\
\operatorname{Hom}\left(\pi_{1}(X), \mathrm{U}(r)\right) \rightarrow \operatorname{Hom}\left(\pi_{1}(C), \mathrm{U}(r)\right) \\
\text { has finite image modulo conjugation }
\end{array}\right) .
$$

The main result of this paper is the construction of such a line bundle $\mathcal{L}_{r}(\S 3)$ with that property (Theorem 4.2). This line bundle $\mathcal{L}_{r}$ is a generalized Theta line bundle. Since we use various facts (see [DN89], [LPo96] and [Pop01]) about these bundles, a short résumé about these objects is presented in $\S \S 3.1-3.4$. In $\S 5$, the line bundle $\mathcal{L}_{r}$ is used to construct a rational version $X \stackrel{\mathrm{alb}_{r}}{\rightarrow} \mathrm{Alb}_{r}(X)$ of a generalized Albanese morphism. This morphism is studied for algebraic surfaces in $\S 6$. We begin this paper by reviewing the constructions of the classical Albanese morphism and describing its fibers in $\S 2$.

In [Kol95], Kollár points out that the Albanese morphism can be regarded as a U(1)-version of the Shafarevich morphism. Hence, our morphism $\mathrm{alb}_{r}$ could be regarded as a $\mathrm{U}(r)$-version of it. Indeed, if the Shafarevich map $X \rightarrow \mathrm{Sh}(X)$ existed, then alb $r$ would factor through it. Katzarkov (see [Kat94] and [Kat97]) defined a reduction morphism for one representation $\pi_{1}(X) \rightarrow \operatorname{GL}(r)$.

Received 22 December 2004, accepted in final form 30 June 2005.

2000 Mathematics Subject Classification 14J10 (primary), 14D20, 14J60 (secondary).

Keywords: Albanese morphism, moduli of vector bundles, generalized Theta divisor.

This journal is (C) Foundation Compositio Mathematica 2006. 


\section{G. HEIN}

In the Kähler case, Eyssidieux [Eys04] showed that $\tilde{X}_{r}$, the universal covering of $X$ modulo those elements of $\pi_{1}(X)$ which are in the kernel of all GL $(r)$ representations, is holomorphically convex.

Notation. We work with schemes over the complex numbers. Since we need the restriction of (semi)stable vector bundles to curves, we are required to use the concept of Mumford-Takemoto or slope stability for vector bundles.

\section{Two constructions for the Albanese variety}

2.1 The classical construction for the Albanese variety. Here we assume that $X$ is a connected Kähler manifold. We define the Albanese variety $\operatorname{Alb}(X)$ to be the quotient

$$
\operatorname{Alb}(X):=H^{0}\left(X, \Omega_{X}^{1}\right)^{\vee} / H_{1}(X, \mathbb{Z}) .
$$

If we choose a point $x_{0} \in X(\mathbb{C})$, then we can define the Albanese morphism $\operatorname{alb}_{X}: X \rightarrow \operatorname{Alb}(X)$ by $x \mapsto \int_{\gamma_{x}}$ where $\gamma_{x}$ is a path connecting $x_{0}$ with $x$.

2.2 The $\operatorname{Pic}^{0}\left(\mathbf{P i c}^{0}\right)$-description of $\mathbf{A l b}(X)$. Let $X$ be a smooth variety over an algebraically closed field $k$. We consider the Picard torus $\operatorname{Pic}^{0}(X)$, i.e. the component of $\operatorname{Pic}(X)$ containing $\mathcal{O}_{X}$. Furthermore, we consider a Poincaré bundle $L$ on $X \times \operatorname{Pic}^{0}(X)$. This bundle is not unique. To normalize it we choose a point $x_{0} \in X(k)$. If we require that $\left.L\right|_{\left\{x_{0}\right\} \times \operatorname{Pic}^{0}(X)} \cong \mathcal{O}_{\operatorname{Pic}^{0}(X)}$, then the Poincaré bundle $L$ is uniquely determined. If we consider $L$ as a family of line bundles on $\operatorname{Pic}^{0}(X)$ parametrized by $X$, then we obtain a morphism from $X$ to the Picard torus of $\operatorname{Pic}^{0}(X)$ :

$$
\operatorname{alb}_{X}: X \rightarrow \operatorname{Pic}^{0}\left(\operatorname{Pic}^{0}(X)\right)=: \operatorname{Alb}(X) .
$$

2.3 Both constructions coincide for smooth varieties over $\operatorname{Spec}(\mathbb{C})$. The use of the same notation in the above constructions is justified, because both coincide for a smooth projective variety over $\operatorname{Spec}(\mathbb{C})$. This follows from the universal property of the Albanese variety and the duality between the Albanese variety and the Picard torus (cf. [GH78]). The following proposition describes the fibers of the Albanese morphism. Since it uses both descriptions ( $\S \S 2.1$ and 2.2), we have to assume that $X / \operatorname{Spec}(\mathbb{C})$ is a smooth projective variety.

Proposition 2.4 (Description of the fibers of the Albanese morphisms alb ${ }_{X}: X \rightarrow \operatorname{Alb}(X)$, for a projective complex manifold $X$ (cf. [GH78, II.6])). If $\iota: Z \rightarrow X$ is a connected cycle in $X$, then the following conditions are equivalent:

(i) $Z$ is contained in a fiber of the morphism $\operatorname{alb}_{X}$;

(ii) the image of $\iota_{*}: H_{1}(Z, \mathbb{Z}) \rightarrow H_{1}(X, \mathbb{Z})$ is finite;

(iii) the pull back morphism $\iota^{*}: H^{0}\left(X, \Omega_{X}^{1}\right) \rightarrow H^{0}\left(Z, \Omega_{Z}^{1}\right)$ is trivial;

(iv) if $\rho: \pi_{1}(X) \rightarrow \mathrm{U}(1)$ is a representation of the fundamental group, then the restriction $\left.\rho\right|_{\pi_{1}(Z)}$ has a finite image;

(v) if $L$ is a line bundle on $X \times S$, then the pull back $\iota^{*} L$ on $Z \times S$ is of the form $L_{1} \otimes L_{2}$, for any Noetherian scheme $S$.

\section{The line bundle $\mathcal{L}_{r}$}

3.1 The generalized Theta divisor, I: The determinant of cohomology. Generalized Theta line bundles play a central role in this paper. Therefore, we repeat their definition, basic properties, as well as the construction of global sections of these line bundles. Let $C$ be a smooth 


\section{Generalized Albanese morphisms}

projective curve of genus $g$. We assume that $\mathcal{E}$ is a vector bundle on $S \times C$ with $S$ a connected Noetherian scheme. We denote the projections as

$$
S \stackrel{q}{\longleftarrow} S \times C \stackrel{p}{\longrightarrow} C .
$$

For a geometric point $s \in S$, we let $\mathcal{E}_{s}$ be the vector bundle $p_{*}\left(\mathcal{E} \otimes q^{*} k(s)\right)$ on $C$. Since $S$ is connected, the rank $r$ and the degree $d$ of $\mathcal{E}_{s}$ do not depend on the choice of $s \in S$. We say that $\mathcal{E}$ is a family of rank $r$ vector bundles of degree $d$ on $C$ parametrized by $S$. If all the vector bundles $\mathcal{E}_{s}$ are semistable, then we obtain a morphism $S \rightarrow M_{C}(r, d)$ to the moduli space of $S$-equivalence classes of semistable vector bundles on $X$. Twisting $\mathcal{E}$ with a line bundle $q^{*} L$ does not change this morphism. Therefore, the families $\mathcal{E}$ and $\mathcal{E} \otimes q^{*} L$ are considered to be equivalent.

For a coherent sheaf $F$ on $C$, we obtain a line bundle $\operatorname{det}\left(q_{!}\left(\mathcal{E} \otimes p^{*} F\right)\right)$ on $S$ by taking the determinant of cohomology. This way, we obtain a morphism from the Grothendieck group $K(C)$ to the Picard group $\operatorname{Pic}(S)$. Since

$$
\operatorname{det}\left(q_{!}\left(\left(\mathcal{E} \otimes q^{*} L\right) \otimes p^{*} F\right)\right) \cong \operatorname{det}\left(q_{!}\left(\mathcal{E} \otimes p^{*} F\right)\right) \otimes L^{\otimes \chi\left(C, F \otimes \mathcal{E}_{s}\right)},
$$

this line bundle is twist invariant whenever $\chi\left(C, F \otimes \mathcal{E}_{s}\right)=0$. Thus, we consider the subgroup $K(C)^{\perp(r, d)}$ of $K(C)$ generated by coherent sheaves $F$ with $\chi\left(C, F \otimes \mathcal{E}_{s}\right)=0$. The main result of Drezet and Narasimhan in [DN89] is that the line bundles $\operatorname{det}\left(q_{!}\left(\mathcal{E} \otimes p^{*} F\right)\right)$ for $F \in K(C)^{\perp(r, d)}$ descend to line bundles on the moduli space $M_{C}(r, d)$, and the resulting morphism $K(C)^{\perp(r, d)} \rightarrow$ $\operatorname{Pic}\left(M_{C}(r, d)\right)$ is surjective.

3.2 The generalized Theta divisor, II: The generalized Theta line bundle. We define the generalized Theta bundle first in the case when $d=0$. We take a vector bundle $F$ of rank 2 and determinant $\omega_{C}$ on $C$. By the Riemann-Roch theorem for curves, we have $[F] \in K(C)^{\perp(r, 0)}$. We denote the generalized Theta line bundle $\mathcal{O}_{S}(\Theta)$ to be the line bundle $\operatorname{det}\left(q_{!}\left(\mathcal{E} \otimes p^{*} F\right)\right)^{-1}$ on $S$.

For $d$ arbitrary, we need a base point $s_{0} \in S$. We choose a vector bundle $F$ of rank $2 r$ and determinant $\omega_{C}^{\otimes r} \otimes \operatorname{det}\left(\mathcal{E}_{S_{0}}\right)^{-\otimes 2}$. Again, we have $[F] \in K(C)^{\perp(r, d)}$ and define $\mathcal{O}_{S}(\Theta)$ to be the line bundle $\operatorname{det}\left(q_{!}\left(\mathcal{E} \otimes p^{*} F\right)\right)^{-1}$ on $S$.

Remark. In both cases, we defined a multiple of the classical generalized Theta divisor. (When $d=0$, we have defined the second power, whereas for $d$ arbitrary, we have defined the $2 \cdot(r, d)$ power of it.) However, to define the appropriate root, further choices are involved.

3.3 The generalized Theta divisor, III: Global sections. $\quad$ Let $\mathcal{O}_{S}(\Theta)=\operatorname{det}\left(q_{!}\left(\mathcal{E} \otimes p^{*} F\right)\right)^{-1}$ be the Theta line bundle and $m$ a natural number. Suppose we are given a finite number of vector bundles $\left\{G_{i}\right\}_{i=1, \ldots, n}$ on $C$ with $[G]=m[F]$ in $K(C)$. The following construction of Le Potier (see [LPo96]) assigns each $G_{i}$ a global section $\theta_{G_{i}}$ in $\Gamma\left(\mathcal{O}_{S}(m \Theta)\right)$. First we take a line bundle $N$ on $C$ such that $G_{i} \otimes N$ is globally generated for all $i$, and $q_{*}\left(\mathcal{E} \otimes p^{*} N^{-1}\right)=0$. Let $R:=\operatorname{rk}\left(G_{i}\right)$. Therefore, we have surjections $\left(N^{-1}\right)^{\oplus(R+1)} \stackrel{\pi_{i}}{\longrightarrow} G_{i}$. The kernel of $\pi_{i}$ is the line bundle $M:=\operatorname{det}\left(G_{i}\right)^{-1} \otimes N^{-\otimes(R+1)}$. Since $\left[G_{i}\right]=\left[G_{j}\right]$ in $K(C)$, the determinant line bundles of $G_{i}$ and $G_{j}$ coincide. Hence, the line bundle $M$ is independent of $i$. From the short exact sequence $0 \rightarrow M \stackrel{\alpha_{i}}{\longrightarrow}\left(N^{-1}\right)^{\oplus(R+1)} \stackrel{\pi_{i}}{\longrightarrow} G_{i} \rightarrow 0$, we pass to the long exact sequence

$$
0 \rightarrow q_{*}\left(\mathcal{E} \otimes p^{*} G_{i}\right) \rightarrow R^{1} q_{*}\left(\mathcal{E} \otimes p^{*} M\right) \stackrel{R^{1}\left(\alpha_{i}\right)}{\longrightarrow} R^{1} q_{*}\left(\mathcal{E} \otimes p^{*}\left(N^{-1}\right)^{\oplus(R+1)}\right) \rightarrow R^{1} q_{*}\left(\mathcal{E} \otimes p^{*} G_{i}\right) \rightarrow 0 .
$$

By construction, $R^{1}\left(\alpha_{i}\right)$ is a homomorphism of vector bundles of the same rank. Thus, we obtain a section

$$
\theta_{G_{i}}:=\operatorname{det}\left(R^{1}\left(\alpha_{i}\right)\right) \in \Gamma\left(\operatorname{det}\left(R^{1} q_{*}\left(\mathcal{E} \otimes p^{*}\left(N^{-1}\right)^{\oplus(R+1)}\right)\right) \otimes\left(R^{1} q_{*}\left(\mathcal{E} \otimes p^{*} M\right)\right)^{-1}\right) .
$$




\section{G. HEIN}

The sections $\theta_{G_{i}}$ are sections of the line bundle $\mathcal{O}_{S}(m \Theta)$. The base change theorem yields that the vanishing loci $V\left(\theta_{G_{i}}\right)$ are given by

$$
V\left(\theta_{G_{i}}\right)=\left\{s \in S \mid H^{*}\left(C, G_{i} \otimes \mathcal{E}_{s}\right) \neq 0\right\} .
$$

3.4 The generalized Theta divisor, IV: Base point freeness. One the one hand, it is obvious that any pair $E$ and $F$ of vector bundles on $C$ with $H^{*}(C, E \otimes F)=0$ is a pair of semistable vector bundles. On the other hand, a result of Popa (see [Pop01]) says that, for any semistable vector bundle $E$ on $C$, and any integer $a \geqslant \operatorname{rk}(E)$, there exists a vector bundle $F$ on $C$ with $\operatorname{rk}(F)=2 a \operatorname{rk}(E)$ and $\operatorname{det}(F)=\omega_{C}^{\otimes a} \otimes \operatorname{det}\left(\mathcal{E}_{s_{0}}\right)^{-\otimes 2 a}$ where $\mathcal{E}_{s_{0}}$ is an arbitrary bundle with the same rank and degree as $E$. Thus, for $a \geqslant r$, the base points of the sections in $\mathcal{O}_{S}(a \Theta)$ described in $\S 3.3$ are the points $s \in S$ parametrizing unstable vector bundles. Drezet and Narasimhan [DN89] showed that the generalized Theta line bundle is an ample line bundle on the moduli space of $S$-equivalence classes of semistable vector bundles. Note that this allows us to distinguish $S$-equivalence classes $\left[E_{1}\right]$ and $\left[E_{2}\right]$ whenever we have a vector bundle $G$ with $H^{*}\left(E_{1} \otimes G\right)=0$ and $H^{*}\left(E_{2} \otimes G\right) \neq 0$.

3.5 The setup. We fix a smooth projective variety $X$ of dimension $n$ with a very ample line bundle $\mathcal{O}_{X}(H)$ and a positive integer $r$. Furthermore, we choose a geometric point $x_{0} \in X$. Let $M_{r}=M_{X}(r, 0,0, \ldots, 0)$ be the moduli space of $S$-equivalence classes of slope semistable rank $r$ bundles $E$ with trivial Chern classes in $H^{*}(X, \mathbb{Z})$. If $E$ is a vector bundle parametrized by $M_{r}$, then we write $[E]$ for the corresponding point in $M_{r}(\mathbb{C})$. By the theorem of Uhlenbeck and Yau (see [UY86]), $M_{r}$ parametrizes flat vector bundles on $X$ or representations of $\pi_{1}(X)$ in $\mathrm{U}(r)$ modulo conjugation. This implies that, for $[E] \in M_{r}$, the restriction $\left.E\right|_{C}$ of $E$ to any curve $C \subset X$ is a semistable vector bundle. This implication is the reason why we have to restrict ourselves to the moduli space of flat vector bundles on $X$. Moreover, $M_{r}$ is a projective scheme provided that we pass to $S$-equivalence classes of semistable bundles. This means we identify any vector bundle $E$ in a short exact sequence $0 \rightarrow E^{\prime} \rightarrow E \rightarrow E^{\prime \prime} \rightarrow 0$ of slope zero bundles with the direct sum $E^{\prime} \oplus E^{\prime \prime}$. Hereafter, we will use the symbol $M_{r}$ (or $\left.M_{r}(X)\right)$ for the projective moduli space of $S$-equivalence classes of slope semistable bundles on $X$.

3.6 The line bundle $\mathcal{O}_{M}\left(D_{H}\right)$. Using the polarization $H$ on $X$, we can define a polarization $D_{H}$ on $M$. We choose a faithfully flat morphism $\psi: \tilde{M} \rightarrow M$ such that we have a universal sheaf $\tilde{E}$ on $\tilde{M} \times X$. This means that, for any point $\tilde{m} \in \tilde{M}$, the sheaf $\tilde{E}_{\tilde{m}}:=\left.\tilde{E}\right|_{\{\tilde{m}\} \times X}$ is a sheaf which belongs to the $S$-equivalence class given by $\psi(\tilde{m})$. The theory of Quot schemes gives the existence of such morphisms. Let $C=H_{1} \cap H_{2} \cap \cdots \cap H_{n-1}$ be a complete intersection of $n-1$ divisors $H_{i} \in|H|$. Furthermore, we take a rank 2 vector bundle $F$ on $C$ with $\operatorname{det}(F) \cong \omega_{C}$. We consider the following morphisms.

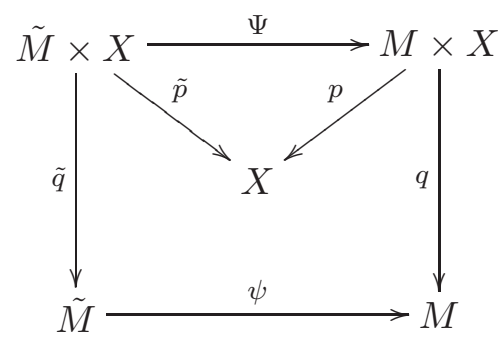

On $\tilde{M}$, we define the line bundle $\mathcal{O}\left(\tilde{D}_{H}\right)$ to be the determinant of cohomology

$$
\mathcal{O}_{\tilde{M}}\left(\tilde{D}_{H}\right):=\operatorname{det}\left(\tilde{q}_{!}\left(\tilde{E} \otimes \tilde{p}^{*} F\right)\right)^{-1} \text {. }
$$

This line bundle descends to $M$, i.e. there exists a line bundle $\mathcal{O}_{M}\left(D_{H}\right)$ and an isomorphism $\mathcal{O}_{\tilde{M}}\left(\tilde{D}_{H}\right) \cong \psi^{*} \mathcal{O}_{M}\left(D_{H}\right)$. Furthermore, the line bundle $\mathcal{O}_{M}\left(D_{H}\right)$ does not depend on the choice of the morphism $\psi: \tilde{M} \rightarrow M$ (see [DN89]). 


\section{Generalized Albanese morphisms}

Lemma 3.7. The first Chern class $\tilde{D}_{H}:=c_{1}\left(\mathcal{O}_{\tilde{M}}\left(\tilde{D}_{H}\right)\right)$ is given by

$$
\tilde{D}_{H}=\tilde{q}_{*}\left(\left(2 c_{2}(\tilde{E})-c_{1}^{2}(\tilde{E})\right) \cdot \tilde{p}^{*} H^{n-1}\right) .
$$

In particular, we have $D_{a H}=a^{n-1} D_{H}$.

Proof. This is straightforward computation using the Grothendieck-Riemann-Roch formula

$$
\tilde{D}_{H}=-\left[\tilde{q}_{*}\left(\operatorname{ch}(\tilde{E}) \cdot \operatorname{Td}(\tilde{q}) \cdot \tilde{p}^{*} \operatorname{ch}(F)\right)\right]_{1},
$$

and the equalities

$$
\begin{array}{lr}
\operatorname{ch}(\tilde{E})=r+c_{1}(\tilde{E})+\frac{c_{1}^{2}(\tilde{E})-2 c_{2}(\tilde{E})}{2}+\cdots, & =1-\frac{\tilde{p}^{*} K_{X}}{2}+\cdots, \\
\operatorname{Td}(\tilde{q})=\tilde{p}^{*} \operatorname{Td}(X) & \\
\operatorname{ch}(F)=\operatorname{ch}\left(\mathcal{O}_{X} \oplus \mathcal{O}_{X}\left(K_{X}+(n-1) H\right)\right) \cdot \operatorname{ch}\left(\mathcal{O}_{H}\right)^{n-1}=2 H^{n-1}+K_{X} \cdot H^{n-1} .
\end{array}
$$

Whereas the first two equalities are standard, the last equality follows from the adjunction formula and the fact that a vector bundle $F$ on a curve $C$ is determined in the Grothendieck group $K(C)$ by its rank and determinant.

Lemma 3.8. The line bundle $\mathcal{O}_{M_{r}}\left(a \cdot D_{H}\right)$ is base point free for $a \geqslant r^{2}$.

Proof. This is just the statement of Popa's theorem given in $\S 3.4$.

Lemma 3.9. The line bundle $\mathcal{O}_{M_{r}}\left(D_{H}\right)$ is ample.

Proof. The proof uses the fact that, on a projective variety of dimension at least two, the vector bundles $E$ and $E^{\prime}$ with the same Hilbert polynomial are isomorphic, if and only if their restrictions to a sufficiently big ample divisor $H$ are isomorphic. This follows from the following long exact sequence.

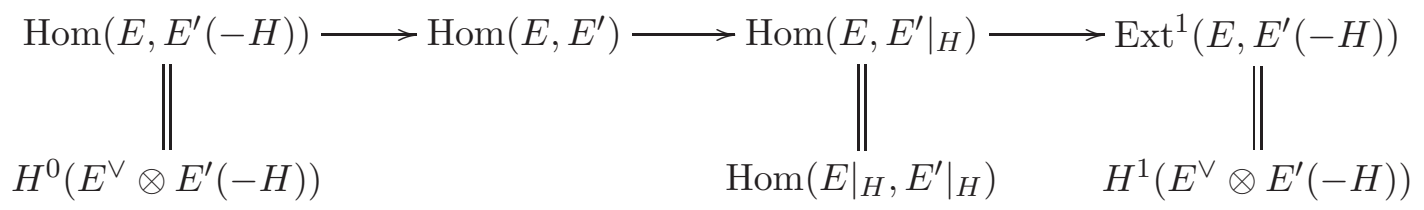

If we have a bounded family of vector bundles as in the case of those parametrized by $M_{r}$, then we can choose a divisor $H$ such that, for any two bundles in this family, the cohomology groups on the left- and right-hand sides vanish.

The restriction theorem of Mehta and Ramanthan (see [MR84]) tells us that, for a semistable vector bundle $E$ and $H$ big enough, the formation of graded objects commutes with restriction to $H$. Thus, we obtain an embedding $M_{r}=M_{r}(X) \longleftrightarrow M_{r}(H)$. Repeating the argument, we end up with an embedding $M_{r} \longleftrightarrow M_{r}(C)$ for a complete intersection curve $C$. By Lemma 3.7, we may assume that this curve $C$ is the curve we considered in the construction of $\mathcal{O}\left(D_{H}\right)$.

By construction, $\mathcal{O}\left(D_{H}\right)$ is the pull back of the generalized Theta line bundle on $M_{r}(C)$. This line bundle is known to be ample by the work of Drezet and Narasimhan (see [DN89]). Thus, the lemma holds.

3.10 The families $\mathcal{E}_{r, i}$. Let $M_{r}^{\text {red }}=\bigcup_{i=1}^{l} M_{r, i}$ be the decomposition of the reduced scheme underlying $M_{r}$ into its irreducible components, and let $\tilde{M}_{r, i}$ be the normalization of the component $M_{r, i}$. We have a morphism $\alpha_{i}: \tilde{M}_{r, i} \rightarrow M_{r}$ and consider the globally generated line bundle (by Lemma 3.8) $N_{r, i}:=\alpha_{i}^{*} \mathcal{O}\left(r^{2} \cdot D_{H}\right)$. Let $C_{r, i}$ be the intersection of $\operatorname{dim}\left(\tilde{M}_{r, i}\right)-1$ general global sections of $N_{r, i}$. By Bertini's theorem, $C_{r, i}$ can be assumed to be a smooth irreducible curve. 


\section{G. HEIN}

Thus, by Langton's theorem (see [Lan75]), we have a universal vector bundle $\mathcal{E}_{r, i}$ on $C_{r, i} \times X$. If the universal vector bundle $\mathcal{E}_{M}$ on $M_{r} \times X$ existed, then $\mathcal{E}_{r, i}$ would be the pull back of this bundle to $C_{r, i} \times X$.

3.11 The line bundle $\mathcal{L}_{r, i}$. We consider the vector bundle $\mathcal{E}_{r, i}$ on $C_{r, i} \times X$ and the morphisms

$$
C_{r, i} \stackrel{q}{\longleftarrow} C_{r, i} \times X \stackrel{p}{\longrightarrow} X .
$$

We define the line bundle $N_{r, i}$ on the curve $C_{r, i}$ by $N_{r, i}:=\operatorname{det}\left(\left.\mathcal{E}_{r, i}\right|_{C_{r, i} \times\left\{x_{0}\right\}}\right)$. Let $G_{r, i}$ be a vector bundle on $C_{r, i}$ with $\operatorname{rk}\left(G_{r, i}\right)=2 r$, and $\operatorname{det}\left(G_{r, i}\right) \cong \omega_{C_{r, i}}^{r} \otimes N_{r, i}^{-2}$. Similar to the definition of $\mathcal{O}_{M_{r}}\left(D_{H}\right)$ in $\S 3.6$, we define the line bundle by

$$
\mathcal{L}_{r, i}:=\operatorname{det}\left(p_{!}\left(\mathcal{E}_{r, i} \otimes q^{*} G_{r, i}\right)\right)^{-1} .
$$

3.12 Remark. Unfortunately, in contrast to $\mathcal{O}_{M_{r}}\left(D_{H}\right)$, the line bundle $\mathcal{L}_{r, i}$ is not independent of the choices. We next give an example for this dependence on the choice of the family $\mathcal{E}_{r, i}$.

Let $X$ be a curve, and $\mathcal{E}_{r, i}$ be a family of degree zero vector bundles on $X$ parametrized by $C_{r, i}$. For a point $c \in C_{r, i}$, we consider the vector bundle $\mathcal{E}_{c}:=\left.\mathcal{E}_{r, i}\right|_{\{c\} \times X}$. Furthermore, we assume that $\mathcal{E}_{c}$ is not stable. Thus, we have a short exact sequence $0 \rightarrow \mathcal{E}_{c}^{\prime} \rightarrow \mathcal{E}_{c} \rightarrow \mathcal{E}_{c}^{\prime \prime} \rightarrow 0$ of degree zero vector bundles on $X$. Denote by $\mathcal{E}_{r, i}^{\prime}$ the kernel of the natural surjection $\mathcal{E}_{r, i} \rightarrow \mathcal{E}_{c}^{\prime \prime}$. The families $\mathcal{E}_{r, i}^{\prime}$ and $\mathcal{E}_{r, i}$ parametrize the same $S$-equivalence classes of vector bundles on $X$. A straightforward computation shows that the resulting line bundles $\mathcal{L}_{r, i}$ and $\mathcal{L}_{r, i}^{\prime}$ fulfill

$$
\mathcal{L}_{r, i}^{\prime}=\operatorname{det}\left(\mathcal{E}_{c}^{\prime}\right)^{2 \operatorname{rk}\left(\mathcal{E}_{c}^{\prime \prime}\right)} \otimes \operatorname{det}\left(\mathcal{E}_{c}^{\prime \prime}\right)^{-2 \operatorname{rk}\left(\mathcal{E}_{c}^{\prime}\right)} \otimes \mathcal{L}_{r, i} .
$$

Thus, we can only hope that the numerical type of $\mathcal{L}_{r, i}$ is well defined. This is the case, as we will see in the next section (see Corollary 4.4).

3.13 We end this section by defining the line bundle $\mathcal{L}_{r}$ on $X$ by

$$
\mathcal{L}_{r}:=\bigotimes_{i=1}^{l} \mathcal{L}_{r, i} .
$$

\section{Properties of the line bundles $\mathcal{L}_{r}$}

4.1 Relations defined by nef line bundles. Let $\mathcal{L}$ be a nef line bundle on a proper variety $X$. This line bundle defines an equivalence relation $\sim_{\mathcal{L}}$ on the geometric points of $X$ as follows:

$$
x \sim_{\mathcal{L}} x^{\prime} \Leftrightarrow\left\{\begin{array}{l}
\text { there exists a closed curve } C \subset X \\
\text { with } x \in C, x^{\prime} \in C, \text { and } \mathcal{L} \cdot C=0
\end{array}\right\} .
$$

We define the relation $\preceq$ on nef line bundles by: the condition $L_{1} \preceq L_{2}$ holds if for any curve $C \subset X$ the inequality $L_{1} . C>0$ implies $L_{2} . C>0$.

We write $L_{1} \prec L_{2}$ if $L_{1} \preceq L_{2}$ holds, and there exists a curve $C \subset X$ with $L_{1} . C=0$ and $L_{2}$. $C>0$. If $L_{1} \preceq L_{2}$ holds, then the relation $\sim_{L_{2}}$ is contained in $\sim_{L_{1}}$, i.e. if $x \sim_{L_{2}} x^{\prime}$, then we have $x \sim_{L_{1}} x^{\prime}$. Whenever both relations $L_{1} \preceq L_{2}$ and $L_{2} \preceq L_{1}$ hold, we write $L_{1} \sim L_{2}$. This means that the relations $\sim_{L_{1}}$ and $\sim_{L_{2}}$ coincide.

If we have a chain $L_{0} \prec L_{1} \prec \cdots \prec L_{k}$ of nef line bundles, then we have strict inclusions $\left(\mathrm{NE}(X) \cap L_{k}^{\perp}\right) \subset\left(\mathrm{NE}(X) \cap L_{k-1}^{\perp}\right) \subset \cdots \subset\left(\mathrm{NE}(X) \cap L_{0}^{\perp}\right)$ in the cone of curves. Since the orthogonal complements $L_{i}^{\perp}$ are linear subspaces of $H^{2}(X, \mathbb{R})$, we deduce that $k \leqslant \rho(X)=\operatorname{dim}(\mathrm{NE}(X))$. 


\section{Generalized Albanese morphisms}

Theorem 4.2. The line bundle $\mathcal{L}_{r, i}$ is nef, i.e. for any morphism $\iota: Y \rightarrow X$ of a smooth curve $Y$ to $X$, the degree of the line bundle $\iota^{*} \mathcal{L}_{r, i}$ is non-negative. Furthermore, if the degree of $\iota^{*} \mathcal{L}_{r, i}$ equals zero, then for all geometric points $y_{1}$ and $y_{2}$ of $Y$ there exists an isomorphism $\mathcal{E}_{C_{r, i} \times\left\{y_{1}\right\}} \cong \mathcal{E}_{C_{r, i} \times\left\{y_{2}\right\}}$, and all the vector bundles on $Y$ parametrized by $C_{r, i}$ are $S$-equivalent.

Proof. We divide the proof into eight steps.

Step 1: Reduction to the case where $X$ is a smooth projective curve. Since all the vector bundles parametrized by $M_{r}$ restrict to semistable bundles on every closed subscheme $\iota: Y \rightarrow X$ of $X$, the pull back $(\mathrm{id} \times \iota)^{*} \mathcal{E}_{r, i}$ is a family of semistable rank $r$ vector bundles on $Y$ parametrized by $C_{r, i}$. Since the determinant of cohomology commutes with base change, we may assume $X=Y$. Thus, we consider the vector bundle $\mathcal{E}_{r, i}$ on the surface $C_{r, i} \times X$ and the following morphisms to smooth curves:

$$
C_{r, i} \stackrel{q}{\longleftarrow} C_{r, i} \times X \stackrel{p}{\longrightarrow} X .
$$

Step 2: The line bundles $L_{1}$ and $L_{2}:=\mathcal{L}_{r, i}$. Let $F$ be a vector bundle on $X$ with $\operatorname{rk}(F)=2 r$ and $\operatorname{det}(F)=\omega_{X}^{r}$. For a point $x_{0} \in X$, we set $N_{r, i}:=\operatorname{det}\left(\left.\mathcal{E}_{r, i}\right|_{C_{r, i} \times\left\{x_{0}\right\}}\right)$. Let $G$ be a vector bundle on $C_{r, i}$ of $\operatorname{rank} 2 r$ with $\operatorname{det}(G)=\omega_{C_{r, i}}^{r} \otimes N_{r, i}^{-2}$. We set $L_{1}:=\operatorname{det}\left(q_{!}\left(\mathcal{E}_{r, i} \otimes p^{*} F\right)\right)^{-1}$ and $L_{2}:=\operatorname{det}\left(p_{!}\left(\mathcal{E}_{r, i} \otimes q^{*} G\right)\right)^{-1}$. The nefness property of $\mathcal{L}_{r, i}$ is equivalent to $\operatorname{deg}\left(L_{2}\right) \geqslant 0$.

The line bundle $L_{2}$ is the generalized Theta line bundle constructed in $\S 3.2$ for the family $\mathcal{E}_{r, i}$ of vector bundles on $C_{r, i}$ parametrized by $X$ with distinguished point $x_{0}$. The line bundle $L_{1}$ is the $r$-fold power of the generalized Theta line bundle from $\S 3.2$ for the family $\mathcal{E}_{r, i}$ of vector bundles of degree zero on $X$ parametrized by $C_{r, i}$.

Step 3: $\operatorname{deg}\left(L_{1}\right)=\operatorname{deg}\left(L_{2}\right)$. We use the Grothendieck-Hirzebruch-Riemann-Roch theorem to compute the degrees of the line bundles $L_{1}$ and $L_{2}$. Let us fix the notation before doing so. By $c_{0}$ and $x_{0}$, we denote two geometric points of $C_{r, i}$ and $X$. We use $F_{p}$ and $F_{q}$ to name the fibers $p^{-1}\left(x_{0}\right)$ and $q^{-1}\left(c_{0}\right)$. The genera of $C_{r, i}$ and $X$ we denote by $g_{C}$ and $g_{X}$. Since we are only interested in the degrees, we may assume $\omega_{X}=\mathcal{O}_{X}\left(\left(2 g_{X}-2\right) x_{0}\right)$ and $\omega_{C_{r, i}}=\mathcal{O}_{X}\left(\left(2 g_{C}-2\right) c_{0}\right)$. For the same reason, we have $\operatorname{ch}(F)=2 r+2 r\left(g_{X}-1\right) x_{0}$ and $\operatorname{ch}(G)=2 r+\left(2 r\left(g_{C}-1\right)-2\left(\int_{C_{r, i} \times X}\left(F_{p} \cdot c_{1}\left(\mathcal{E}_{r, i}\right)\right)\right)\right) c_{0}$. Furthermore, let $\operatorname{Td}\left(C_{r, i}\right)=1-\left(g_{C}-1\right) c_{0}$ and $\operatorname{Td}(X)=1-\left(g_{X}-1\right) x_{0}$ be the (numerical) Todd classes. Then we have

$$
\begin{aligned}
\operatorname{deg}\left(L_{1}\right) & =-\int_{C_{r, i}} \operatorname{ch}\left(q_{!}\left(\mathcal{E}_{r, i} \otimes p^{*} F\right)\right) \\
& =-\int_{C_{r, i} \times X} \operatorname{ch}\left(\mathcal{E}_{r, i} \otimes p^{*} F\right) p^{*} \operatorname{Td}(X) \\
& =-\int_{C_{r, i} \times X} \operatorname{ch}\left(\mathcal{E}_{r, i}\right) p^{*} \operatorname{ch}(F) p^{*} \operatorname{Td}(X) \\
& =-\int_{C_{r, i} \times X} \operatorname{ch}\left(\mathcal{E}_{r, i}\right) p^{*}(\operatorname{ch}(F) \operatorname{Td}(X)) \\
& =-\int_{C_{r, i} \times X}\left(r+c_{1}\left(\mathcal{E}_{r, i}\right)+\frac{c_{1}^{2}\left(\mathcal{E}_{r, i}\right)-2 c_{2}\left(\mathcal{E}_{r, i}\right)}{2}\right) p^{*}(2 r) \\
& =r \cdot \int_{C_{r, i} \times X}\left(2 c_{2}\left(\mathcal{E}_{r, i}\right)-c_{1}^{2}\left(\mathcal{E}_{r, i}\right)\right) .
\end{aligned}
$$




\section{G. HEIN}

Analogously, we obtain for the degree of $L_{2}$ that

$$
\begin{aligned}
\operatorname{deg}\left(L_{2}\right) & =-\int_{C_{r, i} \times X}\left(r+c_{1}\left(\mathcal{E}_{r, i}\right)+\frac{c_{1}^{2}\left(\mathcal{E}_{r, i}\right)-2 c_{2}\left(\mathcal{E}_{r, i}\right)}{2}\right) q^{*}\left(2 r-\left(2 \int_{C_{r, i} \times X}\left(F_{p} \cdot c_{1}\left(\mathcal{E}_{r, i}\right) c_{0}\right)\right)\right) \\
& =r \cdot \int_{C_{r, i} \times X}\left(2 c_{2}\left(\mathcal{E}_{r, i}\right)-c_{1}^{2}\left(\mathcal{E}_{r, i}\right)\right)+2 \cdot\left(\int_{C_{r, i} \times X} F_{p} \cdot c_{1}\left(\mathcal{E}_{r, i}\right)\right) \cdot\left(\int_{C_{r, i} \times X} F_{q} \cdot c_{1}\left(\mathcal{E}_{r, i}\right)\right) .
\end{aligned}
$$

Since $C_{r, i}$ parametrizes a family of $X$ vector bundles of degree zero, the intersection number $\int_{C_{r, i} \times X} F_{q} \cdot c_{1}\left(\mathcal{E}_{r, i}\right)$ equals zero. Thus, we end up with the claimed equality.

Step 4: $L_{1}^{\otimes r}$ is globally generated. Thus, $\operatorname{deg}\left(L_{2}\right)=\operatorname{deg}\left(L_{1}\right) \geqslant 0$. This is a direct consequence of Lemma 3.8.

To prove $\operatorname{deg}\left(L_{1}\right) \geqslant 0$, we need fewer premises. Indeed, if at least one point of $c \in C_{r, i}$ parametrizes a semistable vector bundle $\mathcal{E}_{c}$ on $X$, then a power of $L_{1}$ has a non-trivial section $\theta_{G}$ (see $\S \S 3.3$ and 3.4 ) which is what we need.

If the degree of $L_{1}$ is positive, then we consider two points $s_{1}, s_{2}$ of $C_{r, i}$ with the property that a section $\theta_{G}$ vanishes at $s_{1}$ but not at $s_{2}$. It follows from the remark at the end of $\S 3.4$ that the $X$ vector bundles parametrized by $s_{1}$ and $s_{2}$ are not $S$-equivalent.

From now on, we assume that the degrees of $L_{1}$ and $L_{2}$ are zero. For the following steps see also the proof of Theorem I.4 in Faltings' article [Fal93] or for the simpler rank 2 case see [Hei99, Theorem 3.4].

Step 5: For any two geometric points $P$ and $Q$ of $X$, the vector bundles $\left.\mathcal{E}_{r, i}\right|_{C_{r, i} \times\{P\}}$ and $\left.\mathcal{E}_{r, i}\right|_{C_{r, i} \times\{Q\}}$ are isomorphic. We show that, given a point $P \in X$, for almost all points $Q \in X$, we have an isomorphism between $\left.\mathcal{E}_{r, i}\right|_{C_{r, i} \times\{P\}}$ and $\left.\mathcal{E}_{r, i}\right|_{C_{r, i} \times\{Q\}}$. From this statement, the assertion of step 5 follows immediately. Fix a geometric point $c_{0} \in C_{r, i}$. Let $\mathcal{E}_{c_{0}}:=\left.\mathcal{E}_{r, i}\right|_{\left\{c_{0}\right\} \times X}$ be the semistable vector bundle on $X$ parametrized by $c_{0}$. Take a vector bundle $F_{P}$ on $X$ such that $H^{*}\left(X, F_{P} \otimes \mathcal{E}_{c_{0}}\right)=0$. Such a bundle exists as we have seen in $\S 3.4$. Moreover, it defines a global section $\theta_{F_{P}}$ in a power of $L_{1}$ which does not vanish at $c_{0}$. Since $\operatorname{deg}\left(L_{1}\right)=0$, this section has an empty vanishing divisor. The description of the vanishing divisor of $\theta_{F_{P}}$ (see $\left.\S 3.3\right)$ implies that $H^{*}\left(X,\left.F_{P} \otimes \mathcal{E}_{r, i}\right|_{\{c\} \times X}\right)=0$ for all points $c \in C_{r, i}$. This implies that $R^{*} q_{*}\left(\mathcal{E}_{r, i} \otimes p^{*} F_{P}\right)$ is zero. Now we consider a non-trivial extension $F$ in $\operatorname{Ext}^{1}\left(k(P), F_{P}\right)$ :

$$
\left(\mathcal{S}_{P}\right) \quad 0 \rightarrow F_{P} \rightarrow F \stackrel{\pi_{P}}{\longrightarrow} k(P) \rightarrow 0 .
$$

The scheme $\mathbb{P}(F)$ parametrizes surjections $\pi: F \rightarrow k(Q)$ from $F$ to torsion sheaves of length one. The subset of $\mathbb{P}(F)$ where $H^{*}\left(X, \operatorname{ker}(\pi) \otimes \mathcal{E}_{c_{0}}\right)=0$ is open and not empty because it contains $\pi_{P}$. Thus, for a general point $Q$ of $X$, there exists a short exact sequence

$$
\left(\mathcal{S}_{Q}\right) \quad 0 \rightarrow F_{Q} \rightarrow F \rightarrow k(Q) \rightarrow 0
$$

with $H^{*}\left(X, F_{Q} \otimes \mathcal{E}_{c_{0}}\right)=0$. Applying the functor $R^{*} q_{*}\left(\mathcal{E}_{r, i} \otimes p^{*}(-)\right)$ to the short exact sequences $\left(\mathcal{S}_{P}\right)$ and $\left(\mathcal{S}_{Q}\right)$, we obtain that $q_{*}\left(p^{*} F \otimes \mathcal{E}_{r, i}\right)$ is isomorphic to $\left.\mathcal{E}_{r, i}\right|_{C_{r, i} \times\{P\}}$ and to $\left.\mathcal{E}_{r, i}\right|_{C_{r, i} \times\{Q\}}$ as well.

Thus, all the vector bundles on $C_{r, i}$ parametrized by $X$ are isomorphic to the vector bundle $G:=q_{*}\left(p^{*} F \otimes \mathcal{E}_{r, i}\right)$.

Step 6: The filtration $F^{*}\left(\mathcal{E}_{r, i}\right)$ on the vector bundle $\mathcal{E}_{r, i}$. If we consider the Harder-Narasimhan filtration on $G:=q_{*}\left(p^{*} F \otimes \mathcal{E}\right)$, then the graded summands need not be simple bundles. We consider a slight generalization by taking $G_{1}$ to be a subsheaf of $G$ which is stable of maximal possible slope. Defining $F^{1}\left(\mathcal{E}_{r, i}\right):=G_{1} \otimes p_{*} \mathcal{H} \operatorname{com}\left(q^{*} G_{1}, \mathcal{E}_{r, i}\right)$, and $F^{l}\left(\mathcal{E}_{r, i}\right):=\pi_{1}^{-1}\left(F^{l-1}\left(\mathcal{E}_{r, i} / F^{1}\left(\mathcal{E}_{r, i}\right)\right)\right)$, where $\pi_{1}$ 


\section{Generalized Albanese morphisms}

is the surjection from $\mathcal{E}_{r, i} \rightarrow \mathcal{E}_{r, i} / F^{1}\left(\mathcal{E}_{r, i}\right)$, we obtain a filtration $0=F^{0}\left(\mathcal{E}_{r, i}\right) \subset F^{1}\left(\mathcal{E}_{r, i}\right) \subset \cdots \subset$ $F^{k}\left(\mathcal{E}_{r, i}\right)=\mathcal{E}_{r, i}$ on $\mathcal{E}_{r, i}$ with the property that the $j$ th graded object $\operatorname{gr}^{j}\left(\mathcal{E}_{r, i}\right):=F^{j}\left(\mathcal{E}_{r, i}\right) / F^{j-1}\left(\mathcal{E}_{r, i}\right)$ is of the form $G_{j} \otimes F_{j}$. By definition the slopes $\mu_{j}:=\mu\left(G_{j}\right)=\operatorname{deg}\left(G_{j}\right) / \operatorname{rk}\left(G_{j}\right)$ form a decreasing sequence $\mu_{1} \geqslant \mu_{2} \geqslant \cdots \geqslant \mu_{k}$.

Restricting the filtration $F^{*}\left(\mathcal{E}_{r, i}\right)$ to a fiber $p^{-1}(x)$ of $p$, we obtain a filtration of $G$ which does not depend on the choice of $x \in X$. The restricted vector bundle $\left.F^{j}\left(\mathcal{E}_{r, i}\right)\right|_{p^{-1}(x)}$ appears in the Harder-Narasimhan filtration of $G$, if and only if $\mu_{j}>\mu_{j+1}$. Therefore, we use $\operatorname{HNF}^{*}\left(\mathcal{E}_{r, i}\right)$ to name the subfiltration of the filtration $F^{*}\left(\mathcal{E}_{r, i}\right)$ consisting of those $F^{j}\left(\mathcal{E}_{r, i}\right)$ with $\mu_{j}>\mu_{j+1}$.

Step \%: Numerical invariants of the filtration $F^{*}\left(\mathcal{E}_{r, i}\right)$. In the Grothendieck group $K\left(C_{r, i} \times X\right)$, we can identify $\mathcal{E}_{r, i}$ with the direct sum of the graded objects $\operatorname{gr}^{j}\left(\mathcal{E}_{r, i}\right)$ :

$$
\left[\mathcal{E}_{r, i}\right]=\sum_{j=1}^{k}\left[\operatorname{gr}^{j}\left(\mathcal{E}_{r, i}\right)\right]=\sum_{j=1}^{k}\left[G_{j} \otimes F_{j}\right] .
$$

Since the Chern character of the product $G_{j} \otimes F_{j}$ is given by

$$
\begin{aligned}
\operatorname{ch}\left(G_{j} \otimes F_{j}\right) & =q^{*} \operatorname{ch}\left(G_{j}\right) \cdot p^{*} \operatorname{ch}\left(F_{j}\right) \\
& =\operatorname{rk}\left(G_{j}\right) \cdot \operatorname{rk}\left(F_{j}\right)+\left[\operatorname{rk}\left(G_{j}\right) p^{*} c_{1}\left(F_{j}\right)+\operatorname{rk}\left(F_{j}\right) q^{*} c_{1}\left(G_{j}\right)\right]+p^{*} c_{1}\left(F_{j}\right) \cdot q^{*} c_{1}\left(G_{j}\right),
\end{aligned}
$$

we deduce the equality $\int_{C_{r, i} \times X} \operatorname{ch}\left(\mathcal{E}_{r, i}\right)=\sum_{j=1}^{k} \operatorname{deg}\left(G_{j}\right) \cdot \operatorname{deg}\left(F_{j}\right)$. In step 3, we identified the left hand side with $[-1 /(2 r)] \operatorname{deg}\left(L_{1}\right)$. Thus, we have

$$
\sum_{j=1}^{k} \operatorname{deg}\left(G_{j}\right) \cdot \operatorname{deg}\left(F_{j}\right)=0 .
$$

The degree $\operatorname{deg}_{X}\left(\left.F^{j}\left(\mathcal{E}_{r, i}\right)\right|_{q^{-1}(c)}\right)$ of $F^{j}\left(\mathcal{E}_{r, i}\right)$, restricted to a fiber of $q$, is given by

$$
\operatorname{deg}_{X}\left(\left.F^{j}\left(\mathcal{E}_{r, i}\right)\right|_{q^{-1}(c)}\right)=\sum_{m=1}^{j} \operatorname{rk}\left(G_{m}\right) \cdot \operatorname{deg}\left(F_{m}\right) .
$$

Since the restriction of $\mathcal{E}_{r, i}$ to a fiber of $q$ is semistable of degree zero, we deduce that

$$
A_{j}:=\sum_{m=1}^{j} \operatorname{rk}\left(G_{m}\right) \cdot \operatorname{deg}\left(F_{m}\right) \leqslant 0,
$$

and $A_{k}=0$. Having in mind that $\mu_{j}:=\operatorname{deg}\left(G_{j}\right) / \operatorname{rk}\left(G_{j}\right)$, we rewrite (1) as

$$
0=\sum_{j=1}^{k} \mu_{j} \cdot \operatorname{rk}\left(G_{j}\right) \cdot \operatorname{deg}\left(F_{j}\right)=\sum_{j=1}^{k} A_{j} \cdot\left(\mu_{j}-\mu_{j+1}\right),
$$

where we set $\mu_{k+1}=0$. The inequalities (2) and $\mu_{j} \geqslant \mu_{j+1}$ imply, therefore, that $A_{j}=0$ whenever $\mu_{j}>\mu_{j+1}$.

Step 8: S-equivalence of all bundles parametrized by $C_{r, i}$. The conclusion of the preceding steps is that each quotient $\operatorname{gr}_{\mathrm{HNF}}^{j}:=\operatorname{HNF}^{j}\left(\mathcal{E}_{r, i}\right) / \operatorname{HNF}^{j-1}\left(\mathcal{E}_{r, i}\right)$ is, on the one hand, semistable of degree zero when restricted to the fibers of $q$. On the other hand, when restricting to a fiber of $p$ we obtain a vector bundle which is an extension of stable vector bundles of the same slope. Thus, the restriction of $\operatorname{gr}_{\mathrm{HNF}}^{j}$ to both families of fibers is semistable.

If we construct the Theta line bundle associated to $\mathrm{gr}_{\mathrm{HNF}}^{j}$, then we obtain a line bundle of degree zero on $X$. This follows from the computation in step 7 . Now we have a semistable family of vector bundles parametrized by $X$ with degree zero Theta divisor. The argument of step 5 yields that all the bundles parametrized by the family $\operatorname{gr}_{\mathrm{HNF}}^{j}$ on $X$ are isomorphic. 


\section{G. HEIN}

In other words, the direct sum $\bigoplus_{j=1}^{l} \mathrm{gr}_{\mathrm{HNF}}^{j}$ of the graded objects gives the same direct sum of semistable vector bundles of degree zero on each fiber of $q$. In short, all vector bundles parametrized by $C_{r, i}$ are $S$-equivalent. The proof of Theorem 4.2 is complete.

Theorem 4.3. Let $\iota: Y \rightarrow X$ be a morphism of a smooth curve $Y$ to $X$. We obtain a morphism $\iota_{M_{r, i}}: \tilde{M}_{r, i}(X) \rightarrow M_{r}(Y)$ by the pull back of vector bundles. The following two conditions are equivalent:

(i) the degree of $\iota^{*} \mathcal{L}_{r, i}$ is zero;

(ii) the morphism $\iota_{M_{r, i}}$ maps $\tilde{M}_{r, i}(X)$ to a point.

Proof. We consider the morphisms $C_{r, i} \stackrel{\alpha}{\longrightarrow} \tilde{M}_{r, i}(X) \stackrel{\iota_{M_{r, i}}}{\longrightarrow} M_{r}(Y)$. Theorem 4.2 implies that the degree of $\iota^{*} \mathcal{L}_{r, i}$ is zero, if and only if $\iota_{M_{r, i}}\left(C_{r, i}\right)$ is a point. This implies the theorem because $\tilde{M}_{r, i}(X)$ is irreducible, and $C_{r, i}$ is the intersection of ample divisors.

Corollary 4.4. The equivalence classes of the nef line bundles $\mathcal{L}_{r, i}$ and $\mathcal{L}_{r}$ with respect to $\sim$ (see $\S 4.1$ ) depend neither on the choice of $C_{r, i} \subset \tilde{M}_{r, i}$ nor on the choice of the vector bundle $\mathcal{E}_{r, i}$ on $C_{r, i} \times X$. Furthermore, these equivalence classes are independent of the chosen polarization $H$ on $X$.

Corollary 4.5. C4.5 The line bundle $\mathcal{L}_{r}$ on $X$ is nef. For a morphism $\iota: Y \rightarrow X$ of a smooth curve $Y$ to $X$, we have $\operatorname{deg}\left(\iota^{*} \mathcal{L}_{r}\right)=0$, if and only if the morphism $M_{r}(X) \rightarrow M_{r}(Y)$ is locally constant.

Proposition 4.6. The line bundles $\left\{\mathcal{L}_{r}\right\}_{r \in \mathbb{N}}$ satisfy the inequality $\mathcal{L}_{r_{1}} \preceq \mathcal{L}_{r_{2}}$, for $r_{1} \leqslant r_{2}$. There exists a number $R \in \mathbb{N}$ such that $\mathcal{L}_{r} \preceq \mathcal{L}_{R}$ for all $r$.

Proof. If $r_{1}<r_{2}$, then we have an embedding of moduli spaces $M_{r_{1}} \rightarrow M_{r_{2}}$ given by $[E] \mapsto$ $\left[E \oplus \mathcal{O}_{X}^{\oplus\left(r_{2}-r_{1}\right)}\right]$. Thus, we deduce from Theorem 4.3 that the inequality $\mathcal{L}_{r_{1}} \preceq \mathcal{L}_{r_{2}}$ holds. We have seen in $\S 4$.1 that in the chain $\mathcal{L}_{1} \preceq \mathcal{L}_{2} \preceq \cdots \preceq \mathcal{L}_{r} \preceq \cdots$ there are at most $\rho(X)$ strict inclusions. This proves the second assertion of the proposition.

4.7 The line bundle $\mathcal{L}_{\infty}$. We use the name $\mathcal{L}_{\infty}$ for the line bundle $\mathcal{L}_{R}$ of the above proposition. When referring to this line bundle, we should be aware that $\mathcal{L}_{\infty}$ is only a class in nef line bundles $\} / \sim$. Considering these equivalence classes (with the discrete topology), we have $\lim _{r \rightarrow \infty} \mathcal{L}_{r}$ $\sim \mathcal{L}_{\infty}$.

In the following theorem, we have summarized the results of this section.

Theorem 4.8 (Properties of the line bundles $\mathcal{L}_{r}$ ). Let $X$ be a projective variety. We have an infinite sequence of nef line bundles $\mathcal{L}_{1} \preceq \mathcal{L}_{2} \preceq \cdots \preceq \mathcal{L}_{r} \preceq \cdots$ and a nef limit line bundle $\mathcal{L}_{\infty}$ with $\lim _{r \rightarrow \infty} \mathcal{L}_{r} \sim \mathcal{L}_{\infty}$ on $X$ such that for any morphism $\iota: Y \rightarrow X$ of a smooth curve $Y$ to $X$ the following conditions are equivalent:

(i) $\operatorname{deg}\left(\iota^{*} \mathcal{L}_{r}\right)=0$;

(ii) the restriction morphism $M_{r}(X) \rightarrow M_{r}(Y)$ is locally constant;

(iii) for any connected scheme $Z$ and every vector bundle $\mathcal{E}$ on $Z \times X$ parametrizing semistable rank $r$ vector bundles on $X$ with trivial Chern classes, the pull back $\left(\operatorname{id}_{Z} \times \iota\right)^{*} \mathcal{E}$ parametrizes only one $S$-equivalence class on $Y$;

(iv) modulo conjugation only finitely many $\mathrm{U}(r)$ representations of $\pi_{1}(Y)$ are induced by those of $\pi_{1}(X)$. 


\section{Generalized Albanese morphisms}

\section{The generalized Albanese morphisms}

5.1 The construction of the generalized Albanese morphism. If $\mathcal{L}_{r}$ or some power of it were base point free, then it would define a morphism $\psi: X \rightarrow \mathbb{P}^{m}$. Let $\varphi: X \rightarrow \operatorname{Alb}_{r}$ be the Stein factorization of $\psi$, i.e. $\varphi$ is surjective with connected fibers. Two geometric points $x$ and $x^{\prime}$ of $X$ have the same image under $\varphi$, if and only if $x \sim_{\mathcal{L}_{r}} x^{\prime}$. By Theorem 4.8 , the map $\varphi$ would meet the requirements of a generalized Albanese variety. Indeed, a curve $Y \stackrel{\iota}{\rightarrow} X$ would be contracted by $\varphi$, if $\operatorname{deg}_{Y}\left(\iota^{*} \mathcal{L}_{r}\right)=0$. This means (by Theorem 4.8) that all families of semistable rank $r$ vector bundles on $X$ with trivial Chern classes become constant when restricted to $Y$, or only finitely many representation classes modulo conjugation of $\pi_{1}(Y)$ in $\mathrm{U}(r)$ are induced by representations of $\pi_{1}(X)$.

If no line bundle $L_{r}$ with $L_{r} \sim \mathcal{L}_{r}$ is base point free (note that $L^{\otimes k} \sim L$, for all $k>0$ ), then Tsuji's nef reduction theorem provides us with a rational version of the generalized Albanese variety up to birational equivalence. In this case we obtain only a birational model of the Albanese morphism and variety.

TheOrem 5.2 (see [BCE02, Theorem 2.1], see also [Tsu00]). There exists a dominant rational map $X \stackrel{\mathrm{alb}_{r}}{\rightarrow} \operatorname{Alb}_{r}(X)$ with connected fibers such that:

(i) the line bundle $\mathcal{L}_{r}$ is numerically trivial on all compact fibers $F$ of $\operatorname{alb}_{r}$ of dimension $\operatorname{dim}(X)-$ $\operatorname{dim}\left(\operatorname{Alb}_{r}(X)\right)$;

(ii) for every general point $x \in X$ and every irreducible curve $C$ passing through $x$ with $\operatorname{dim}\left(\operatorname{alb}_{r}(C)\right)>0$, we have C.. $\mathcal{L}_{r}>0$;

(iii) there exist compact fibers of $\mathrm{alb}_{r}$.

Furthermore, the pair $\left(\mathrm{alb}_{r}, \mathrm{Alb}_{r}(X)\right)$ is uniquely determined up to birational equivalence.

5.3 The chain of generalized Albanese morphisms. Even though we end up with an infinite sequence of rational morphisms $X \stackrel{\operatorname{alb}_{r}}{\rightarrow} \operatorname{Alb}_{r}(X)$, for each $r \in \mathbb{N}$, there are at most $\rho(X)+1$ different generalized Albanese morphisms, since for almost all $r \in \mathbb{N}$, we have $\mathcal{L}_{r} \sim \mathcal{L}_{r+1}$. Since $\mathcal{L}_{r} \preceq \mathcal{L}_{r+1}$, we get a rational morphism $\operatorname{Alb}_{r+1}(X)-->\operatorname{Alb}_{r}(X)$. So, we end up with the following commutative diagram.

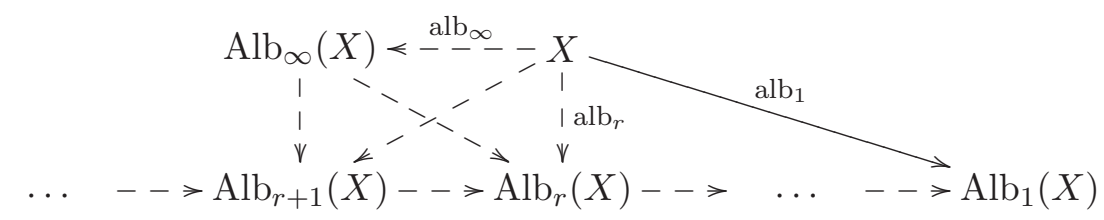

Proposition 5.4 (Functoriality). If $\psi: X \rightarrow X^{\prime}$ is a morphism of projective varieties, then we have $\psi^{*} \mathcal{L}_{r}^{\prime} \preceq \mathcal{L}_{r}$ for all $r \in \mathbb{N} \cup\{\infty\}$. Therefore, we have the following commutative diagram.

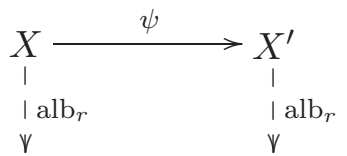

$$
\operatorname{Alb}_{r}(X)-->\operatorname{Alb}_{r}\left(X^{\prime}\right)
$$

Proof. Suppose that $\iota: Y \rightarrow X$ is a morphism from a smooth curve to $X$ with $\operatorname{deg}\left(\iota^{*} \psi^{*} \mathcal{L}_{r}^{\prime}\right)>0$. This implies by Theorem 4.3 that there exists a family $\mathcal{E}^{\prime}$ of rank $r$ vector bundles with trivial Chern classes on $X^{\prime}$ parametrized by a connected scheme $Z$ such that the pull back $\left(\operatorname{id}_{Z} \times(\psi \circ \iota)\right)^{*} \mathcal{E}^{\prime}$ of $\mathcal{E}^{\prime}$ to $Z \times Y$ parametrizes different $S$-equivalence classes on $Y$. However, then the family $\mathcal{E}=\left(\mathrm{id}_{Z} \times \psi\right)^{*}$ also parametrizes rank $r$ vector bundles on $X$ which pull back to non- $S$-equivalent classes. Consequently, again by Theorem $4.3, \operatorname{deg}\left(\iota^{*} \mathcal{L}_{r}\right)>0$. 


\section{G. HEIN}

Proposition 5.5. If $\psi: X \rightarrow X^{\prime}$ is an étale morphism of projective varieties, then we have $\mathcal{L}_{r} \preceq \psi^{*} \mathcal{L}_{r \cdot \operatorname{deg}(\psi)}^{\prime}$ for all $r \in \mathbb{N}$. Furthermore, $\mathcal{L}_{\infty} \sim \psi^{*} \mathcal{L}_{\infty}^{\prime}$.

Proof. The proof is analogous to the preceding one. We simply have to consider the push forward of a family of rank $r$ vector bundles on $X$ to $X^{\prime}$. The statement about $\mathcal{L}_{\infty}$ is easily obtained from $\mathcal{L}_{r} \preceq \psi^{*} \mathcal{L}_{r \cdot \operatorname{deg}(\psi)}^{\prime} \preceq \mathcal{L}_{r \cdot \operatorname{deg}(\psi)}$ and the fact that $\mathcal{L}_{r} \sim \mathcal{L}_{\infty}$ for $r \gg 0$.

5.6 Remark. Since the first Albanese variety and morphism exist, it would be enough to have the base point freeness of some power of $\mathcal{L}_{r}$ on every fiber of the Albanese variety to obtain the following regular morphism.

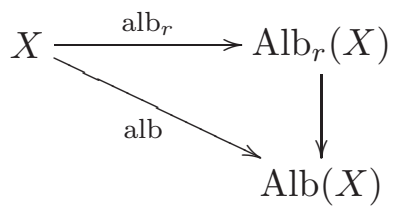

This is used to study the generalized Albanese morphisms in the case of algebraic surfaces in the next section.

\section{The case of algebraic surfaces}

6.1 We consider here the case of a polarized projective algebraic surface $(X, H)$. In this case, we can make a much stronger statement than Theorem 5.2. The generalized Albanese morphism is well understood if $X$ is not of general type (see $\S 6.9$ ). The rest of this section is devoted to the proof of the following theorem.

Theorem 6.2. Let $(X, H)$ be a polarized projective surface. Then there exists a surjective morphism $\operatorname{alb}_{r}: X \rightarrow \operatorname{Alb}_{r}(X)$ with connected fibers, and an effective divisor $D$ on $X$, such that for all morphisms $\iota: C \rightarrow X$ of irreducible curves with $\iota(C) \not \subset D$ the following conditions are equivalent:

(i) $\operatorname{alb}_{r}(\iota(C))$ is a point;

(ii) the associated morphism $\operatorname{Hom}\left(\pi_{1}(X), \mathrm{U}(r)\right) \stackrel{\iota^{*}}{\longrightarrow} \operatorname{Hom}\left(\pi_{1}(C), \mathrm{U}(r)\right)$ modulo conjugation has a finite image;

(iii) for any base scheme $S$ and any rank $r$ vector bundle $E$ on $X \times S$ such that, for each $s, E_{s}$ is semistable with numerically trivial Chern classes, the pull back of $E$ to $C \times S$ is a family of S-equivalent vector bundles.

The divisor $D$ of exceptions can be written in the form $D=C_{1}+C_{2}+\cdots+C_{l}$, where the $C_{i}$ are irreducible and form a basis of a proper subspace of the rational Néron-Severi vector space $\mathrm{NS}(X) \otimes \mathbb{Q}$. In particular, we have $l<\operatorname{dim}_{\mathbb{Q}}(\mathrm{NS}(X) \otimes \mathbb{Q})$.

6.3 Preparations for the proof. We consider the nef line bundle $\mathcal{L}_{r}$ on $X$ satisfying the equivalence of Theorem 4.3. There are two extreme cases where the proof is a simple remark. When $C \cdot \mathcal{L}_{r}>0$, for all curves $C$, then we set $\mathrm{alb}_{r}$ to be the identity morphism of $X$. If $C$. $\mathcal{L}_{r}=0$ for all curves, then we set $\operatorname{Alb}_{r}(X)=\operatorname{Spec}(\mathbb{C})$, and we are finished.

Thus, we assume from now on that $\mathcal{L}_{r}$ is a numerically non-trivial nef line bundle vanishing on a non-empty set $\left\{C_{i}\right\}_{i \in I}$ of irreducible curves. It follows from the construction that all the curves $C_{i}$ in this family are contracted to points by the classical Albanese morphism.

Being a nef divisor, $\mathcal{L}_{r}$ is the limit of ample divisors (with rational coefficients), which yields $\mathcal{L}_{r}^{2} \geqslant 0$. This implies the following lemma.

Lemma 6.4. If $C \subset X$ is an effective divisor with $C^{2}>0$, then $C$. $\mathcal{L}_{r}>0$. 


\section{Generalized Albanese morphisms}

Proof. We assume the contrary. Since $\mathcal{L}_{r}$ is nef this means that $C . \mathcal{L}_{r}=0$. The Hodge index theorem (see [BPV84, IV.2.15]) implies that $\mathcal{L}_{r}^{2} \leqslant 0$ with equality only when $\mathcal{L}_{r}$ is torsion. From $\mathcal{L}_{r}^{2} \geqslant 0$ and the assumption that $\mathcal{L}_{r}$ is numerically non-trivial we derive a contradiction.

The proof of Theorem 6.2 is subdivided into three cases depending on the dimension of $X$ in the Albanese variety. This is by definition the dimension of $\operatorname{Alb}_{1}(X)$.

6.5 The nef reduction for $\operatorname{Alb}_{1}(X)=\operatorname{Spec}(\mathbb{C})$. We consider the curves $\left\{C_{i}\right\}_{i \in I}$ as vectors in the rational Nerón-Severi space $\mathrm{NS}_{\mathbb{Q}}(X)$. The dimension of this vector space is the Picard number $\rho(X)$ of $X$. The points $\left\{C_{i}\right\}_{i \in I}$ lie on the hyperplane $\left\{C \in \mathrm{NS}_{\mathbb{Q}}(X) \mid C \cdot \mathcal{L}_{r}=0\right\}$. Suppose that there is a non-trivial linear relation among the $C_{i}$. If the number of these curves exceeds $\rho(X)-1$, then we have at least one such relation. We write the linear relation $a_{1} C_{1}+\cdots+a_{m} C_{m}=a_{m+1} C_{m+1}+$ $\cdots+a_{M} C_{M}$ with positive rational $a_{i}$, and $C_{i}$ different from $C_{j}$ whenever $i \neq j$.

After multiplication with a positive integer, we may assume the $a_{i}$ to be integers. We set $D_{1}:=$ $a_{1} C_{1}+\cdots+a_{m} C_{m}$ and $D_{2}:=a_{m+1} C_{m+1}+\cdots+a_{M} C_{M}$. Since $D_{1}$ and $D_{2}$ coincide in $\operatorname{NS}_{\mathbb{Q}}(X)$, their difference is torsion in the Nerón-Severi group. So again, after multiplying with an integer, we may assume that the effective Cartier divisor classes $D_{1}$ and $D_{2}$ coincide. (Here we use the fact that the Picard torus, the dual of the Albanese torus, is trivial.)

Because $D_{1}$ and $D_{2}$ have no common components, $D_{1}^{2}=D_{1} \cdot D_{2} \geqslant 0$. In view of Lemma 6.4 , and $D_{1} \cdot \mathcal{L}_{r}=0$, we conclude that $D_{1}^{2}=0$. This implies that $D_{1}$ and $D_{2}$ are disjoint.

Consequently, the line bundle $L:=\mathcal{O}_{X}\left(D_{1}\right) \cong \mathcal{O}_{X}\left(D_{2}\right)$ has two linearly independent sections which do not intersect. Thus, $L$ is base point free and defines a morphism whose Stein factorization we denote by $\operatorname{alb}_{r}: X \rightarrow \operatorname{Alb}_{r}(X)$.

On the one hand, we have that $F \cdot \mathcal{L}_{r}=0$ for all fibers of alb $_{r}$. On the other hand, suppose $C . \mathcal{L}_{r}=0$ for a curve $C \subset X$. Let $F$ be an irreducible fiber of $\operatorname{alb}_{r}(X)$. If $C$ were not contained in a fiber, then we would have $(C+m F)^{2}>0$ for $m \gg 0$. However, we have $(C+m F) \cdot \mathcal{L}_{r}=0$, which contradicts Lemma 6.4. Thus, each curve $C$ with $C \cdot \mathcal{L}_{r}=0$ is contained in a fiber.

This means that the effective divisor $D$ of Theorem 6.2 can be taken to be the empty set once we have a linear relation between the $\left\{C_{i}\right\}_{i \in I}$ in $\mathrm{NS}_{\mathbb{Q}}(X)$. Since the resulting morphism alb $\mathrm{b}_{r}$ contracts all these curves, we conclude that $\operatorname{alb}_{r}$ does not depend on the chosen linear relation.

6.6 The nef reduction when $\operatorname{Alb}_{1}(X)$ is a curve. We consider the morphism alb $1: X \rightarrow$ $\mathrm{Alb}_{1}(X)$. This morphism is the Stein factorization of the classical Albanese morphism. It follows from $\mathcal{L}_{1} \preceq \mathcal{L}_{r}$ that each curve $C$ with $C$. $\mathcal{L}_{r}=0$ is contained in a fiber of this morphism. Let $F$ be the generic fiber of alb 1 .

If $F \cdot \mathcal{L}_{r}=0$, then all curves $C$ with $C \cdot \mathcal{L}_{r}=0$ are contracted by alb 1 . Consequently we set $\mathrm{alb}_{r}=\mathrm{alb}_{1}$ and Theorem 6.2 is proven.

We suppose now that F. $\mathcal{L}_{r}$ is positive. The set of curves $\left\{C_{i}\right\}_{i \in I}$ consists of components of reducible fibers of the morphism alb. We will show that this set is not only finite but linearly independent in $\mathrm{NS}_{\mathbb{Q}}(X)$. Indeed, if there were a linear relation, then we would obtain (see $\left.\S 6.5\right)$ an effective divisor $D_{1}=a_{1} C_{1}+\cdots+a_{m} C_{m}$. This divisor satisfies $D_{1}^{2}=0, D_{1} \cdot \mathcal{L}_{r}=0$, and consists of fiber components. This contradicts Zariski's lemma (see [BPV84, Lemma III.8.2]), because $F . \mathcal{L}_{r}>0$ for all fibers $F$. This shows that Theorem 6.2 holds when setting $\operatorname{alb}_{r}=\mathrm{id}_{X}$.

6.7 Remark. The finite collection of curves $\left\{C_{i}\right\}_{i \in I}$ must have a negative definite intersection matrix, because of Zariski's lemma. Thus, by Grauert's criterion (see [BPV84, Theorem III.2.1]), there exists a contraction of these curves. However, this contraction is not necessarily a projective morphism. If it were, we could take this contraction to be our generalized Albanese morphism alb . $_{\text {. }}$ 


\section{G. HEIN}

6.8 The nef reduction for $\operatorname{dim}\left(\mathrm{Alb}_{1}(X)\right)=2$. In this case, the generalized Albanese morphism alb $1: X \rightarrow \operatorname{Alb}_{1}(X)$ contracts finitely many curves. Among those curves are the $\left\{C_{i}\right\}_{i \in I}$ which are numerically trivial with respect to $\mathcal{L}_{r}$. The intersection matrix of these $\left\{C_{i}\right\}_{i \in I}$ is negative definite. This yields that these curves form a basis of a proper subspace of $\mathrm{NS}_{\mathbb{Q}}(X)$. Again, just setting $\mathrm{alb}_{r}=\mathrm{id}_{X}$, the assertions of Theorem 6.2 are fulfilled. As before, $\S 6.7$ applies.

6.9 Surfaces of Kodaira dimension less than 2. Let $X$ be a projective algebraic surface of Kodaira dimension $\kappa(X) \leqslant 1$. We assume that $X$ is minimal. This is not a restriction, because the fundamental group of rational curves is zero. Table 1 gives the generalized Albanese morphism for these surfaces following the Enriques-Kodaira classification (see [BPV84, VI]). The sixth and seventh rows are perhaps the most interesting ones. They show that the generalized Albanese morphisms may reveal more of the surface than the classical one. We assume in these two rows that the surface $X$ is not a product of two curves.

TABle 1. Surfaces $X$ with $\kappa(X) \leqslant 1$.

\begin{tabular}{lll}
\hline$\kappa(X)$ & class of $X$ & the generalized Albanese morphism \\
$-\infty$ & rational surfaces & $X \rightarrow \operatorname{Spec}(\mathbb{C})$ \\
$-\infty$ & ruled surfaces $X \rightarrow B$ with $g(B) \geqslant 1$ & $X \rightarrow B$ \\
0 & Enriques surfaces & $X \rightarrow \operatorname{Spec}(\mathbb{C})$ \\
0 & K3 surfaces & $X \rightarrow \operatorname{Spec}(\mathbb{C})$ \\
0 & tori & $X \rightarrow X$ \\
0 & hyperelliptic surfaces & $\operatorname{Alb}_{1}(X)$ is an elliptic curve, whereas \\
& & $\operatorname{Alb}_{r}(X) \cong X$, for $r \gg 1$ (see also $\S 6.10$ ) \\
1 & properly elliptic surfaces with & $\operatorname{Alb}_{1}(X)$ is an algebraic curve, and \\
& smooth elliptic fibration & $\operatorname{Alb}_{r}(X) \cong X$, for $r \gg 1$ (see also $\left.\S 6.10\right)$ \\
1 & properly elliptic surfaces with only & $\operatorname{alb}_{r}: X \rightarrow \operatorname{Alb}(X)$ is the elliptic \\
& generically smooth elliptic fibration & fibration for all $r \in \mathbb{N}$ \\
\hline
\end{tabular}

6.10 A class of examples. Let $G$ be a finite group with $|G|$ elements and $C_{1}, C_{2}$ be two smooth projective curves with a $G$ action such that:

(a) the genera $g_{C_{1}}$ and $g_{C_{2}}$ are positive;

(b) $G$ acts free on $C_{1}$, i.e. the quotient map $C_{1} \rightarrow C_{1} / G$ is étale;

(c) there are no $G$-invariant global sections in $H^{0}\left(C_{2}, \omega_{C_{2}}\right)$, which is equivalent to $C_{2} / G \cong \mathbb{P}^{1}$.

We obtain a free $G$-action on $C_{1} \times C_{2}$. Let $X:=\left(C_{1} \times C_{2}\right) / G$ be the quotient of this action and $p: C_{1} \times C_{2} \rightarrow X$ the projection. Since $C_{1} \times C_{2}$ is embedded into its Albanese variety, we deduce from Proposition 5.5 that $\mathcal{L}_{|G|} . C>0$ for all curves $C \subset X$. Thus, $\operatorname{alb}_{r}=\operatorname{id}_{X}: X \rightarrow X$ for all $r \geqslant|G|$, whereas the classical Albanese morphism is just the map to the curve $C_{1} / G$.

\section{ACKNOWLEDGEMENT}

The author wishes to thank the referee for valuable proposals and corrections. 


\section{Generalized Albanese morphisms}

\section{REFERENCES}

BPV84 W. Barth, C. Peters and A. Van de Ven, Compact complex surfaces (Springer, Berlin, 1984).

BCE02 Th. Bauer, F. Campana, Th. Eckl, S. Kebekus, Th. Peternell, S. Rams, T. Szemberg and L. Wotzlaw, A reduction map for nef line bundles, in Complex geometry, collection of papers dedicated to Hans Grauert, eds I. Bauer, F. Catanese, Y. Kawamata, T. Peternell and Y.-T. Siu (Springer, Berlin, 2002), 27-36.

DN89 J.-M. Drezet and M. S. Narasimhan, Groupe de Picard des variétés de modules de fibrés semi-stables sur les courbes algébriques, Invent. Math. 97 (1989), 53-94.

Eys04 P. Eyssidieux, Sur la convexité holomorphe des revêtements linéaires réductifs d'une variété projective algébrique complexe, Invent. Math. 156 (2004), 503-564.

Fal93 G. Faltings, Stable G-bundles and projective connections, J. Algebraic Geom. 2 (1993), 507-568.

GH78 P. Griffiths and J. Harris, Principles of algebraic geometry, Pure and Applied Mathematics (Wiley, New York, 1978).

Hei99 G. Hein, Duality construction of moduli spaces, Geom. Dedicata 75 (1999), 101-113.

Kat94 L. Katzarkov, Factorization theorems for the representations of the fundamental groups of quasiprojective varieties and some applications, Preprint (1994), arXiv:math.AG/9402012.

Kat97 L. Katzarkov, On the Shafarevich maps, Proc. Sympos. Pure Math., vol. 62, part 2 (American Mathematical Society, Providence, RI, 1997), 173-216.

Kol95 J. Kollár, Shafarevich maps and automorphic forms (Princeton University Press, Princeton, NJ, 1995).

Lan75 S. Langton, Valuative criteria for families of vector bundles on algebraic varieties, Ann. of Math. (2) 101 (1975), 88-110.

LPo96 J. Le Potier, Module des fibrés semistables et fonctions thêta, in Proc. symp. Taniguchi, Kyoto, 1994: Moduli of vector bundles, ed. M. Maruyama, Lecture Notes in Pure and Applied Mathematics, vol. 179 (Dekker, New York, 1996), 83-101.

MR84 V. B. Mehta and A. Ramanathan, Restriction of stable sheaves and representations of the fundamental group, Invent. Math. 77 (1984), 163-172.

Pop01 M. Popa, Dimension estimates for Hilbert schemes and effective base point freeness on moduli spaces of vector bundles, Duke Math. J. 107 (2001), 469-495.

Tsu00 H. Tsuji, Numerically trivial fibrations, Preprint (2000), arXiv:math.AG/0001023.

UY86 K. Uhlenbeck and S.-T. Yau, On the existence of Hermitian Yang-Mills connections on stable vector bundles I, Comm. Pure Appl. Math. 39 (1986), 257-293; II, Comm. Pure Appl. Math. 42 (1989) 703-707.

Georg Hein ghein@math.fu-berlin.de

Freie Universität Berlin, Fachbereich Mathematik und Informatik, Institut für Mathematik II, Arnimallee 3, D-14195 Berlin, Germany 